\title{
EDITORIAL
}

\section{Undulant Fever, Melitococemia and focalizations, Brucellosis to be rediscovered}

Since, advent of $20^{\text {th }}$ Century, Brucellosis has been making smoldering impact on health care system in developed as well as developing countries.

The places dependent on livestock, inherited rural customs, social taboos and sub-optional immunization schedule make transmission of brucellosis rapid.

Brucellosis mainly a zoonotic disease makes transmission from animal kingdom to humans through breach of good preventive guidelines.

It presents mainly as Undulant fever, which characterizes itself as remitting and relapsing pattern. Its other name of Malta fever derive from its location in Portugal, Israel and Jordan.

As the organism, which is a cocci bacilli gains entry into blood and melitococemia is produced incapacitating an individual to the level of prostration. It is confirmed by culturing the blood in tryptose medium or albini medium.

As disease progresses and not treated it localizes in various organs to limit their physiology. It can form abscess in liver, spleen, myocardium or muscle. It can produce endo-carditis or lead to spondodiscitis.

Due to slow growth of bacteria, more often than not the cultures are unrewarding. It can take days to week for optional growth of or gain on the conventional media. Though present day liquid medium like BACTEC has promising outcome.

Sometimes ethinic practices like milk drawing from rudder in semi preventive societies help nurture brucellosis but advanced societies where contaminated ice cream and barbeques help disseminate infection.

High risk professions like butchers, abbatiors, vets, farmers, health care workers etc are always source of dispersion.

Middle Eastern countries find brucella endemic for variety of reasons. A myth cultivated over many centuries that raw camel milk energize sexual desires has found a place among males.

Despite availability of serology, culture and PCR at some centres still clinical diagnosis remains the centre spread for institution of antibiotics for the brucella. Amasing fact for the brucella remains, that it can be acute severe in presentation to chronic insidious ill health. Human organs like heart, spleen, liver, brainetc remain sanctuary areas for brucellosis. Traditional antibiotic like aminoglycocides, rifampicin and tetracycles are corner store of management. Some anti bacterial activity is seen with beta lactams, TMPSMX and quinolones. For acceptable log kill to be achieved we always need combination of drugs.

Future strategies lies in prevention, vaccination, early diagnosis and appropriate drugs with appropriate dosage and duration. Many countries like Australia, Greece, USA, UK have been declared Brucella free since 1985 but active surveillance of cattle, their products, humans is being performed periodically.

The article, refers to the grim outlook of vetenary and medical practice in Nepal. The authors are apprehensive of a great toll brucella is going to take on health care practice in their country.

The preventive and curative practices in India as well as Kashmir are no different. Brucella remains a health care issue in our place as well.

A word of caution at the end needs to be mentioned, brucella from point of biologic warfare. It remains a threat as a medium for bioterrorism in future.

Dr. Ajaz Nabi Koul MD, MRCP (UK), MRCP (London), FCAN, FRCPE

\section{REFERENCES:}

1. Lim ML, Richman LS. Brucellosis Inf Dis Clinics 2004; 12:7-14.

2. Herrick J.A. et al., Brucellosis Clinical Manifestation treatment and prognosis lancet Inf. Disease 2014; 4(6): 5206 